\title{
COMPLICAÇÕES DECORRENTES DO TRATAMENTO DIALÍTICO: CONTRIBUIÇÃO DO ENFERMEIRO NO CUIDADO E EDUCAÇÃO AO PACIENTE
}

\author{
COMPLICATIONS ARISING FROM DIALYTIC TREATMENT: \\ CONTRIBUTION OF NURSES IN CARE AND PATIENT EDUCATION
}

\section{Heloisa Helena Lemos Horta', Mara Lisa Lopes ${ }^{2}$}

'Enfermeira. Mestre em Promoção da Saúde. Docente na Universidade de Franca. Franca, São Paulo, Brasil. helohorta@yahoo.com.br ${ }^{2}$ Enfermeira. Graduada pela Universidade de Franca. Franca, São Paulo, Brasil. maralisa_lopes@yahoo.com

RESUMO I A doença renal crônica (DRC) é a incapacidade dos rins eliminarem substâncias tóxicas e manter o equilíbrio hidroeletrolítico. $O$ paciente com DRC vive grandes dificuldades durante o tratamento dialítico, especificamente a hemodiálise. Durante $\circ$ período de tratamento ele enfrenta uma avalanche de mudanças em sua vida, psicológica, física, familiar e social. O objetivo deste estudo visa conhecer as complicações sofridas pelo paciente renal crônico submetido à terapia dialítica e as intervenções do enfermeiro no cuidado e educação desse cliente. $O$ método utilizado trata-se de um estudo em língua portuguesa, de caráter exploratório, com pesquisa na base de dados Scielo, em livros na área de nefrologia, bem como pesquisa eletrônica na internet. A literatura aponta que os pacientes com DRC encontram dificuldades no tratamento dialítico como falta de conhecimento da doença e seus sintomas, desconhecimento do tratamento, vindo a ter reflexo no ambiente familiar, psicológico e social. Tais dificuldades podem mudar com a intervenção do enfermeiro por ser o profissional mais próximo e constante desse paciente, através de medidas educativas ao paciente e familiar.

Descritores: Doença renal crônica; Hemodiálise; Educação em saúde.

\begin{abstract}
Chronic kidney disease (CKD) is the inability of the kidneys to eliminate toxic substances and maintain hydroelectrolyte balance. The CKD patient experiences great difficulties during dialysis, specifically hemodialysis. During the treatment period he faces an avalanche of changes in his life, psychological, physically, family and social. The purpose of this study is to know the complications suffered by the chronic renal patient submitted to dialytic therapy and the interventions of the nurse in the care and education of this client. The method used is a study in Portuguese, of exploratory nature, with research in the Scielo database, in books in the area of nephrology, as well as electronic research on the internet. The literature shows that patients with CKD have difficulty in dialytic treatment, such as a lack of knowledge of the disease and its symptoms, lack of treatment, and a reflex in the family, psychological and social environment. Such difficulties can change with the intervention of the nurse because he is a closer and constant professional of this patient, through educational measures to the patient and family.
\end{abstract}

Keywords: Chronic kidney disease; Hemodialysis; Health education. 


\section{INTRODUÇÃO}

A DRC consiste na perda progressiva e irreversível da função renal, quando os rins não são capazes de manter o equilíbrio hidroeletrolítico e metabólico do organismo. O paciente apresenta hipertensão, anorexia, vômito, alterações no nível de consciência, agitação, dor e desconforto intenso'.

Atualmente a DRC é uma doença que se tornou um problema de Saúde Pública, diante do aumento significativo em prevalência e incidência. Quando não é tratada, e ou não controlada, evolui para doença renal terminal, responsável pelo aumento da morbimortalidade, necessitando de terapia renal substitutiva ${ }^{2}$.

O tratamento deve ser imediato e por tempo indeterminado a fim de controlar a uremia e evitar a morte do paciente. A uremia é caracterizada por alterações clínicas e metabólicas como resistência a ação da insulina, inflamação, acidose metabólica, anemia, distúrbios do metabolismo ósseo e gastrintestinal ${ }^{3,4}$.

No Brasil o método mais empregado para o tratamento da DRC é a hemodiálise, que remove substâncias nitrogenadas tóxicas e excesso de água através de um circuito extracorpóreo formado por linha arterial e venosa de material sintético e um hemodialisador.

A fístula arteriovenosa (FAV) é considerada como acesso "padrão ouro" da hemodiálise. Quando o paciente necessita dialisar antes da fistula estar pronta, o procedimento é realizado através de cateter central de duplo lúmen ${ }^{3,10}$.

A FAV é o acesso mais adequado por ser de longa permanência e por garantir menor número de intervenções. Sua confecção é feita cirurgicamente através de uma anastomose subcutânea permitindo fluxo sanguíneo direto da artéria para veia ${ }^{5,6}$.

Outra modalidade de tratamento é a diálise peritoneal, caracterizada pela utilização da membrana peritoneal como meio de troca para difusão de solutos urêmicos e ultrafiltração de líquidos excedente no organismo. É usado um cateter flexível que é implantado cirurgicamente no abdome. Quando esta solução entra em contato com a membrana peritoneal remove escorias sanguíneas indesejadas e água ${ }^{7}$.

O paciente com DRC apresenta limitações físicas para realizar atividades, andar, realizar esforços físicos, carregar peso, dentre outras, gerando tristeza devido às adaptações e às mudanças geradas, e essas mudanças não acontecem de maneira rápida, é um processo complexo ${ }^{8}$.

O enfermeiro é o profissional mais constante e próximo desse paciente, está constantemente fornecendo cuidados de enfermagem e intervenções educativas visando ajudá-lo a conviver com as novas mudanças e sobreviver com a doença'.

O objetivo deste estudo visa conhecer as complicações sofridas pelo paciente renal crônico submetido à terapia dialítica e as intervenções do enfermeiro no cuidado e educação desse cliente.

\section{MATERIAL E MÉTODOS}

Trata-se de um estudo de revisão da literatura de caráter exploratório com pesquisas na base de dados Scielo, em livros na área de nefrologia, bem como pesquisa eletrônica na internet, realizada no período de agosto de 2014 a dezembro de 2015. Foi utilizado como critério de inclusão artigos na língua portuguesa, na íntegra, com assuntos direcionados ao tema, publicados entre 2009 a 2015. Os descritores utilizados foram: doença renal crônica, hemodiálise e educação em saúde. Foram encontradas 48 publicações, das quais 28 foram excluídas por não se adequarem aos critérios de inclusão propostos e 20 foram selecionadas, além de um livro da Biblioteca da Universidade de Franca, uma dissertação de doutorado e uma monografia.

\section{Revisão de Literatura}

A doença renal é caracterizada quando os rins perdem suas funções regulatórias, excretórias e endócrinas e pode ser de forma aguda ou crônica. 
Doença renal aguda se define como perda da função renal de forma súbita, após horas ou dias, associada a outras doenças graves, provocando acúmulo de substâncias nitrogenadas como ureia e creatinina, podendo estar acompanhada ou não de diminuição de diurese, a princípio, com grandes chances do paciente se recuperar sem alterações de outros órgãos ${ }^{10}$.

A doença renal crônica apresenta lesão, com perda lenta, progressiva e irreversível da função renal e em sua fase mais avançada os rins perdem a capacidade de manter $\circ$ equilíbrio metabólico e hidroeletrolítico, afetando o funcionamento de outros órgãos ${ }^{10}$.

Diversas doenças podem causar a DRC, entre elas estão a nefropatia diabética, nefropatia hipertensiva, as glomerulonefrites crônicas e doença renal policística, sendo as mais frequentes, ocasionando alterações hematológicas, endócrinas, neurológicas, gastrintestinais, dermatológicas e hidroeletrolíticas ${ }^{2}$.

\section{A Sociedade Brasileira de Nefrologia (SBN)} classifica a DRC em seis estágios: estágiol agrupase os indivíduos que estão na faixa de risco para insuficiência renal, como diabéticos, hipertensos, histórico de DRC na família, com taxa de filtração glomerular (TFG) igual ou maior a $90 \mathrm{~mL} /$ $\mathrm{min} / 1,73 \mathrm{~m}^{2}$; estágio 2 pacientes com lesão, mas, com função renal normal e TFG de 60 e $89 \mathrm{~mL} /$ $\mathrm{min} / 1,73 \mathrm{~m}^{2}$; estágio 3 pacientes apresentando insuficiência renal funcional ou leve, detectada por avaliação funcional eficiente, TFG igual a 45 a 59 $\mathrm{mL} / \mathrm{min} . / 1,73 \mathrm{~m}^{2}$; estágio 4 , por meio de exames laboratoriais observa-se alterações nos níveis de creatinina e ureia, TFG igual a 30 a $44 \mathrm{~mL} /$ $\min$./ $1,73 \mathrm{~m}^{2}$; estágio 5 : insuficiência renal clínica ou severa, uremia, sintomas digestivos, fraqueza, mal estar, anemia, edema e hipertensão arterial, TFG igual a 15 a $29 \mathrm{~mL} / \mathrm{min} . / 1,73 \mathrm{~m}^{2}$ e estágio 6 : pacientes com insuficiência renal crônica terminal, perda da função renal, TFG menor que $15 \mathrm{~mL} /$ $\min . / 1,73 \mathrm{~m}^{2}{ }^{10}$.

A DRC é geralmente assintomática e requer acompanhamento rigoroso no seu estágio inicial, implicando na sua prevenção e controle. $O$ tratamento conservador ou pré-dialítico são conjuntos de medidas com $\circ$ objetivo de diminuir - ritmo de progressão da doença e auxiliar na melhora das condições clínicas, físicas e psicológicas das pessoas acometidas ${ }^{11}$.

$\mathrm{Na}$ doença renal, as funções hemostáticas ficam comprometidas e ocorrem rápidas e graves anormalidades no volume ou na composição dos líquidos corporais, podendo ocorrer acúmulo de potássio, ácidos, líquidos e outras substâncias que podem causar a morte do paciente em poucos dias, sendo necessário neste caso, intervenções como hemodiálise para manter o equilíbrio hidroeletrolítico do paciente ${ }^{12}$.

Tais manifestações ocorrem de forma progressiva levando à DRC terminal, necessitando de terapia renal substitutiva, podendo ser a hemodiálise, diálise peritoneal ou transplante. Nesta fase, do diagnóstico até o início da terapia, o paciente apresenta vários sintomas desde náuseas e vômitos, como também, pode apresentar emergências clínicas como edema pulmonar agudo. É comum o paciente notar alterações no sono, memória e o aparecimento de lentificação neurológica. Os tratamentos de diálise peritoneal e hemodiálise não são curativos, apenas paliativos, aliviam alguns sintomas da doença e preservam a vida do paciente substituindo algumas funções renais ${ }^{2,13}$.

A diálise peritoneal é uma terapia mais simples que proporciona menores complicações ao paciente e permite maior autonomia e liberdade, podendo ser feita em domicílio. É caracterizada pela introdução de uma substância salina com dextrose na cavidade peritoneal através de um cateter, sendo o de Tenckhoff o mais usado. A solução entra em contato com a membrana peritoneal e promove a retirada de substâncias tóxicas do sangue, através de trocas periódicas da solução de diálise ${ }^{14,15}$.

A hemodiálise é um processo de filtração para eliminar principalmente a ureia e a creatinina da corrente sanguínea. Ocorre nesse processo, a transferência dessas toxinas do sangue para a solução de diálise (dialisato) por meio de uma membrana semipermeável artificial ${ }^{13}$.

Para a hemodiálise é necessário um equipamento cujo sistema de circulação extracorpórea é 
impulsionado por uma bomba, onde o encontro do sangue com $\circ$ dialisato é feito por difusão. $O$ tratamento tem duração de 3 a 4 horas, realizado de 3 a 4 vezes por semana, pelo resto da vida ou até que aconteça um transplante bem-sucedido ${ }^{1,16}$.

Este equipamento, máquina hemodialisadora de alta complexidade, permite várias funções, tais como: aquecer, avaliar e fornecer a solução de diálise, impulsionando o sangue através de uma bomba e monitorando vários parâmetros do processo (pressão do sangue, pressão do dialisato, fluxo do sangue, fluxo do dialisato, concentração do dialisato, temperatura, presença de ar no compartimento sanguíneo, presença de sangue no dialisato e volume de ultrafiltração). Uma série de alarmes ligados evita acidentes e torna $\circ$ processo bastante seguro ${ }^{17}$.

O paciente com DRC convive diariamente com uma doença incurável e de longa duração. Com a evolução da doença, surgem complicações a curto e longo prazo que provocam limitações e alterações que repercutem na vida do doente e de seus familiares.

O excesso de sódio e água favorece a hipertensão arterial, devendo o paciente ter comparado seu peso atual com $\circ$ peso ideal, além de ser administrado anti-hipertensivo para controlar as crises hipertensivas ${ }^{3}$.

Atenção também com as cãimbras musculares, resultantes da retirada de líquido em excesso, causando diminuição do volume intravascular e da perfusão muscular, sendo adequado prolongar o tempo de diálise, tornando a ultrafiltração menos intensa, além da administração de solução de glicose ou solução hipertônica a fim de aumentar a perfusão para os músculos. É importante orientar - paciente quanto ao controle do ganho de peso interdialítico. Outra complicação é a síndrome do desequilíbrio da diálise, causada pela demora na transferência de ureia do cérebro para o sangue, causando um gradiente na concentração que favorece a translocação de água para $\circ$ interior das células do sistema nervoso central causando edema cerebral, náuseas, vômitos, prurido, cefaleia, febre e calafrios e o enfermeiro deve estar apto para intervir ${ }^{3}$.
Com uma rotina de difícil adaptação o paciente se torna desanimado e desesperado, sendo a depressão o problema psicológico mais importante, podendo progredir para suicídio ou interrupção do tratamento, fazendo-se necessário encorajar esse paciente e estimular suas capacidades para que se adapte ao seu novo estilo de vida de maneira positiva?.

Para melhor adaptação, o diagnóstico precoce da DRC é fundamental, uma vez que a descoberta tardia se deve à falta de conhecimento da população acerca da prevenção e também da pouca informação oferecida pelos profissionais de saúde na atenção em saúde. Sendo assim, o trabalho do enfermeiro no processo de educação nas unidades de saúde do SUS (Sistema Único de Saúde) é de extrema importância devido a sua proximidade com os usuários, atuando na prevenção de várias doenças crônicas, como a doença renal ${ }^{b}$.

Estudos informam que pacientes submetidos à diálise, enfrentam perdas e alterações da imagem e das funções orgânicas, sendo necessário que - enfermeiro conheça estratégias de adesão ao tratamento, pois estas informações serão a base para a enfermagem intervir na adaptação do paciente. Orientações para $\circ$ autocuidado e prática de exercícios físicos, são estratégias que causam maior motivação ao tratamento, através da aquisição de hábitos saudáveis ${ }^{18,19}$.

De acordo com a SOBEN (Associação de Enfermagem em Nefrologia) fundada em 1983, as competências do enfermeiro são agrupadas em: administrativa, assistencial, educativa e de pesquisa. A respeito das competências educativas, esse processo é direcionado aos clientes e familiares e está relacionada à qualidade de vida, podendo ser efetuada por meio de orientações e formulação de manuais educativos adequados à realidade social do cliente ${ }^{20}$. As ações de educação em saúde realizada pelo enfermeiro, conscientizando a população da importância da prevenção, pode evitar que a DRC seja descoberta quando estiver já bem avançada 6 .

Quanto a ingestão de potássio o enfermeiro deve orientar $\circ$ paciente a evitar 0 consumo de frutas e hortaliças que concentrem teor elevado deste 
elemento. Já na hiperfosfatemia, a orientação baseia-se no uso de quelantes de fósforo e educação alimentar desaconselhando $\circ$ paciente a consumir alimentos ricos em fósforo como miúdos, refrigerantes a base de cola, cerveja, chocolates, oleaginosas e alimentos industrializados que contenham como conservante o ácido fosfórico. A restrição de sódio visa o controle da pressão arterial, devendo ser evitado alimentos processados (embutidos, enlatados, condimentos e industrializados), além da utilização mínima de sal no preparo da alimentação, evitando também o sal light por conter alto teor de potássio?.

Para o paciente anúrico, recomenda se a ingestão de $500 \mathrm{ml}$ de líquidos em 24 horas, e diante da falta de clareza no que é considerado líquido, deve ser enfatizado que café, chá, sopa, água de coco, sorvete, frutas e legumes com muita água devem der incluídos neste total ingerido. A falta de compreensão sobre as necessidades de restrição de sódio e líquidos se refletem no desequilíbrio hídrico do paciente, podendo levá-lo ao edema agudo de pulmão e até a morte?

Outro aspecto importante a ser trabalhado neste processo dee ducação, a fim de minimizar complicações com os acessos vasculares é o planejamento de cuidados, orientações de autocuidados, e técnicas adequadas ao manuseio dos acessos vasculares pela equipe de enfermagem ${ }^{21}$.

A fístula arteriovenosa (FAV), anastomose entre uma artéria e uma veia, é de grande importância ao paciente, considerando que o acesso venoso é prioridade para o tratamento hemodialítico. Após sua confecção é necessário esperar geralmente um período de 4 a 6 semanas para iniciar as punções, tempo necessário para que ocorra uma dilatação venosa suficiente com o objetivo de permitir um fluxo de sangue adequado ${ }^{22}$.

O paciente deve ser orientado quanto aos cuidados com a FAV, tais como: não verificar pressão arterial nem coletar sangue no membro com a fístula, realizar exercício de compressão manual para maturação do acesso, evitar usar roupas apertadas e evitar carregar peso ou deitar sobre o braço, palpar o acesso para verificar presença de frêmito (sensação de vibração), lavar o braço do acesso com água e sabão neutro ou antisséptico antes da punção na unidade de hemodiálise, retirar curativo após 4 a 6 horas, proteger de traumatismos, aplicar compressas frias por 24 horas no local após punção, procurar a equipe de nefrologia se identificar anormalidades no acesso e no membro do acesso ${ }^{21}$.

Visto a tamanha responsabilidade do enfermeiro frente o paciente com doença renal crônica, se faz necessário constante atualização profissional, considerando que a comunicação com o paciente para a educação em saúde deve ser realizada dia a dia. Essa comunicação entre enfermeiro e paciente é chamada de comunicação terapêutica, que tem a finalidade de identificar e atender as necessidades de saúde do paciente e contribuir para a melhora da prática de enfermagem, despertando oportunidades e confiança nos pacientes, permitindo que eles sintam satisfação e segurança em todas as fases do tratamento ${ }^{1,23}$.

\section{CONCLUSÃO}

São muitas as dificuldades enfrentadas pelo paciente com DRC em tratamento hemodialítico. As limitações, restrições, negação ao tratamento, depressão e tantas outras complicações que também ocorrem durante a sessão de diálise, deixam o paciente vulnerável e temeroso diante das novas mudanças em sua vida.

O enfermeiro é o profissional mais presente durante o tratamento do paciente com doença renal crônica. Sua participação em todas as etapas vividas por este paciente permite conhecer, avaliar, atuar nos cuidados, intervir e preparar ações educativas para proporcionar melhor qualidade de vida. Este profissional preparado deverá investir na orientação a esses pacientes o mais precoce possível com a finalidade de que seu tratamento tenha $\circ$ mínimo de complicações, capacitando-os quanto ao autocuidado, promovendo assim situação de bemestar mesmo em meio a tantas limitações. 


\section{CONFLITOS DE INTERESSES}

Nenhum conflito financeiro, legal ou político envolvendo terceiros (governo, empresas e fundações privadas, etc.) foi declarado para nenhum aspecto do trabalho submetido (incluindo mas não limitandose a subvenções e financiamentos, conselho consultivo, desenho de estudo, preparação de manuscrito, análise estatística, etc).

\section{REFERÊNCIAS}

1. Pereira MRS, Bispo AO, Ramalho LP, Teixeira SLSP, Rodrigues JA. Papeis da enfermagem na hemodiálise. Rebes. $2013 ; 3(2): 26-36$.

2. Titan S. Principios básicos de nefrologia. Porto Alegre: Artmed; 2013.

3. Sancho POS, Tavares RP, Lago CCL. Assistência de enfermagem frente as principais complicações do tratamento hemodialítico em pacientes renais crônicos. Rev. Enfermagem Contemporânea. 2013;2(1):169-183. doi: 10.17267/23173378rec.v2i2.302

4. Barbosa TBC, Mecenas AS, Barreto JG, Silva MIB, Bregman $R$, Avesani $C M$. Avaliação longitudinal do estado nutricional de pacientes com doença renal crônica na fase não-dialítica. Ceres 2010;5(3):127-137.

5. Pessoa NRC, Linhares FMP. Pacientes em hemodiálise com fistula arteriovenosa: conhecimento, atitude e prática. Esc. Anna Nery. 2015;19(1):73-79. doi: 10.5935/14148145.20150010

6. da Silva AS, da Silveira RS, Fernandes GFM, Lunardi $\mathrm{VL}$, Backes VMS. Percepções e mudança na qualidade de vida de pacientes submetidos a hemodiálise. Rev. bras. enferm. 2011 ;64(5):839-44. doi: 10.1590/S003471672011000500006

7. dos Santos FK, Valadares GV. Conhecendo as estratégias de ação e interação utilizadas pelos clientes para o enfrentamento da diálise peritoneal. Esc Anna Nery. 2013;17(3):423-431. doi: 10.1590/S1414$\underline{81452013000300004}$

8. dos Santos I, Rocha RPF, Berardinelli LMM. Qualidade de vida de clientes em hemodiálise e necessidades de orientação de enfermagem para o autocuidado. Esc. Anna Nery. 2011 ; 15(1):31-38. doi: 10.1590/S1414$\underline{81452011000100005}$

9. dos Santos I, Rocha RPF, Berardinelli LMM. Necessidades de orientação de enfermagem para o autocuidado de clientes em terapias de hemodiálise. Rev. Bras. Enfermagem. 2011 ;64(2):335-42. doi: 10.1590/S003471672011000200018
10. Siviero P, Machado CJ, Rodrigues RN. Doença renal crônica: um agravo de proporções crescentes na população Brasileira. Cedeplar [Internet]. 2013 [acesso em: 09 jan. 2015]. Disponível em: http://www.cedeplar.ufmg.br/ pesquisas/td/TD\%20467.pdf.

11. Roso CC, Beuter M, Jacobi CS, da Silva CT, Perrando MS, Bruinsma JL. Progressão da insuficiência renal crônica: percepções de pessoas em pré-diálise. Rev. Enfermagem UFSM. 2013;3(Esp.):581-588. doi: $\underline{10.5902 / 2179769211020}$

12. Medeiros AJS, de Medeiros EMD. A assistência de enfermagem prestada no tratamento hemodialítico promovido junto ao portador de insuficiência renal crônica. Rebes. $2013 ; 3(2): 13-17$.

13. Cassini AV, Malagutti W, Rodrigues FSM, de Deus RB, Barnabe AS, Francisco L, et al. Avaliação dos principais fatores etiológicos em indivíduos portadores de insuficiência renal crônica em hemodiálise. Conscientiae Saúde. 2010;9(3):462-468.

14. Furtado AMO, de Souza SROS, Oliveira BL, Garcia CN. $O$ enfermeiro assistencial e educador em uma unidade de transplante renal: uma questão desafiadora. Enfermeria Global. $2012.27(5): 351-355$.

15. Calderan C, Torres AAP, Zilmer JGV, Schwartz E, da Silva DGV. Práticas de autocuidado de pessoas com insuficiência renal crônica submetidas a dialise peritoneal ambulatorial continua. R. pesq.: cuid. fundam. 2013. 5(1):3394-02. doi: $10.9789 / 2175-5361.2013 v 5 n 1 p 3394$

16. Batista MGJ. Aceitação da doença crônica: um estudo no adulto em tratamento de hemodiálise. [tese de doutorado]. Porto Alegre: Faculdade de medicina da universidade do porto; 2011.

17. de Deus BPM, Hoerb A, Zanon RB, Morares PS, Agra HC. Sintomas e complicações agudas relacionadas com a hemodiálise. Rev. Epidemiol. Control. Infect. 2015;5(1):52-56. doi: $\underline{10.17058 / \text { reci.v5i1.4951 }}$

18. Madeiro AC, Machado PDLC, Bonfim IM, Braqueais $A R$, Lima FET. Adesão de portadores de insuficiência renal crônica ao tratamento de hemodiálise. Acta Paul Enfermagem. 2010;23(4):546-51. doi: 10.1590/S0103$\underline{21002010000400016}$

19. Dipp T, da Silva VG, Baumgartem MC, Sturmer G, Plentz RDM. Intervenções interdisciplinares no cuidado ao paciente com doença renal crônica em hemodiálise. Rev. Extendere. 2013;2(1):10-22.

20. de Oliveira NB, Silva FVC, Assad LG. Competências do enfermeiro especialista em nefrologia. Rev. Enfermagem UFRJ. 2015;23(3):375-80. doi: $10.12957 /$ reveri.2015.9789 
21. Bobsin C. Fatores intervenientes na auto-higienizaçao do braço antes da punção de pacientes em hemodiálise no hospital de clínicas de Porto Alegre [monografia]. Porto Alegre: Universidade Federal do Rio Grande do Sul; 2009.

22. Fernandes EFS, Soares W, Santos TC, Moriya TM, Terçariol CAS, Ferreira V. Fístula arteriovenosa: autocuidado em enfermagem com doença renal crônica. Rev. Medicina.

$2013 ; 46(4): 424-8$

23. de Oliveira AM, Soares E. A comunicação como importante ferramenta na orientação em unidade de hemodiálise: um estudo reflexivo. Saúde e transf. Soc. 2014;5(3)118-123. 